\title{
Absence of association between whole blood viscosity and delirium after cardiac surgery: a case-controlled study
}

Shokoufeh CheheiliSobbi 1,2,3*, Mark van den Boogaard', Arjen J. C. Slooter ${ }^{4}$, Henry A. van Swieten², Linda Ceelen ${ }^{1}$, Gheorghe Pop ${ }^{3}$, Wilson F. Abdo ${ }^{1}$ and Peter Pickkers ${ }^{1}$

\begin{abstract}
Background: Delirium after cardiothoracic surgery is common and associated with impaired outcomes. Although several mechanisms have been proposed (including changes in cerebral perfusion), the pathophysiology of postoperative delirium remains unclear. Blood viscosity is related to cerebral perfusion and thereby might contribute to the development of delirium after cardiothoracic surgery. The aim of this study was to investigate whether whole blood viscosity differs between cardiothoracic surgery patients with and without delirium.

Methods: In this observational study postoperative whole blood viscosity of patients that developed delirium (cases) were compared with non-delirious cardiothoracic surgery patients (controls). Cases were matched with the controls, yielding a 1:4 case-control study. Serial hematocrit, fibrinogen, and whole blood viscosity were determined pre-operatively and at each postoperative day. Delirium was assessed using the validated Confusion Assessment Method for the Intensive Care Unit or Delirium Screening Observation scale.

Results: In total 80 cardiothoracic surgery patients were screened of whom 12 delirious and 48 matched non-delirious patients were included. No significant difference was found between both groups in fibrinogen $(p=0.36)$, hematocrit $(p=0.23)$ and the area under curve of the whole blood viscosity between shear rates 0.02 and $50 s^{-1}(p=0.80)$ or between shear rates 0.02 and $5 s^{-1}(p=0.78)$.

Conclusion: In this case control study in cardiothoracic surgery patients changes in whole blood viscosity were not associated with the development of delirium.
\end{abstract}

Keywords: Cardiothoracic surgery, Delirium, Intensive care, Medicine, Whole blood viscosity

\section{Background}

Delirium is a serious neuropsychiatric disorder characterized by an acute onset of altered mental status, hallmarked by difficulty in sustaining attention with typically a fluctuating course [1]. Delirium occurs frequently in hospitalized patients, especially in Intensive Care Unit (ICU) patients [2]. In cardiothoracic surgery the incidence of delirium during the postoperative on the ICU is reported to be between $13 \%$ and $42 \%[3,4]$. Postoperative delirium in cardiothoracic surgery patients

\footnotetext{
* Correspondence: Shokoufeh.CheheiliSobbi@radboudumc.nl

'Department of Intensive Care Medicine, Radboudumc, Nijmegen, The Netherlands

${ }^{2}$ Department of Cardiothoracic Surgery, Radboudumc, Nijmegen, The Netherlands

Full list of author information is available at the end of the article
}

is associated with increased length of ICU and hospital stay, increased risk of sternal wound infection, unwanted removal of arterial/venous lines or epicardial electrodes, significantly impaired quality of life and higher longterm morbidity, mortality and healthcare costs $[5,6]$.

The pathophysiological mechanism of delirium is far from clear [7]. Apart from other possible pathways related to the development of delirium [8], reduced cerebral blood flow during delirium with normalization during recovery has been reported $[9,10]$. Cerebral blood flow is strongly related to whole blood viscosity (WBV) [11]. Changes in blood viscosity occur postcardiothoracic surgery [12]. As such, changes in blood viscosity could relate to occurrence of postoperative 
delirium and this could represent an important interventional target to prevent or treat delirium.

Blood viscosity is higher at low shear rates, e.g. in the microcirculation [13]. Therefore, an increased WBV leads to a larger reduction in microcirculatory blood flow compared to blood flow in larger blood vessels. Since cellular perfusion is dependent on microcirculatory flow [14] and blood viscosity affects microcirculatory flow, we hypothesized that changes in viscosity could be related to the development of delirium. The aim of our study was to investigate whether whole blood viscosity differs between cardiothoracic surgery patients with and without delirium.

\section{Methods}

\section{Study design and patients}

This is an exploratory, matched, case-control study carried out in the Radboud University Medical Center, Nijmegen, The Netherlands. Annually approximately 1000 cardiothoracic patients are operated in the RadboudUMC. This study was approved by the medical ethical committee of Arnhem-Nijmegen (study number 2012/ 297) which waived the need for informed consent.

The study population consisted of patients of 50 years or older after an elective cardiothoracic surgical onpump procedure for coronary artery bypass grafting (CABG) or single heart valve surgery. For purpose of homogeneity of the total group, patients who underwent CABG combined with valve surgery were not included. Other exclusion criteria were the use of blood cardioplegia, since this is associated with the development of postoperative neurological events [15], preoperative use of heparin, since heparin could decrease the blood viscosity [16], extracorporeal circulation (ECC) time exceeding $120 \mathrm{~min}$, because ECC time is associated with neurological injury [17], or inability to screen for delirium.

Patients developing delirium postoperatively were defined as cases, and patients in which no delirium occurred served as non-cases. The group of cases was matched $1: 4$ to controls to increase the power of the study. Matching was performed on several important preoperative and postoperative risk factors for the development of delirium [7]: gender, age, duration of surgery, aortic cross clamp (AOX) time, ECC time, severity of illness score (Acute Physiology and Chronic Health Evaluation (APACHE)-II score), and risk of death after a heart operation (European System for Cardiac Operative Risk Evaluation (Euro score)).

\section{Delirium screening}

Delirium assessment was performed three times a day. In order to obtain maximal sensitivity and specificity we used a three way approach to diagnose delirium. Firstly, in the ICU the most specific and sensitive scoring test for delirium, the validated Confusion Assessment Method for the ICU (CAM-ICU), was used by trained ICU nurses [18]. Secondly, for the non-ICU patients, on the cardiothoracic surgical ward, nurses used the validated Delirium Screening Observation (DOS) scale [19]. Unfortunately some patients are not diagnosed by validated tests [20]. Therefore, to not miss these patients nursing and medical files were screened for signs of delirium as the last approach [21]. Delirium was defined as having a positive CAM-ICU score, or a DOS scale $\geq 3$. In order to maximize the sensitivity of the diagnosis delirium, we also checked the medical records when haloperidol was administered for treatment combined with delirium signs noted in the nursing or medical files in case of a negative CAM-ICU score.

\section{Data collection and variables}

Earlier studies show that changes in blood viscosity occurs immediately after induction of anesthesia, immediately after surgery, 1 and 2 days after surgery and normalizes between 3 and 4 days post-cardiothoracic surgery [12]. For this reason blood samples were collected at four time points: preoperatively, directly after the induction of anesthesia $\left(T_{-1}\right)$, within one hour of ICU admission $\left(\mathrm{T}_{0}\right)$, day one $\left(\mathrm{T}_{24}\right)$ and three days $\left(\mathrm{T}_{72}\right)$ after cardiothoracic surgery. Blood was drawn from the central venous catheter. If this was not possible, blood was taken from an indwelling arterial catheter or by vena puncture. During each blood collection the most important determinants of WBV, hematocrit and serum fibrinogen, were also measured and taken into consideration during viscosity calculations [11, 22]. Also presence of diabetes mellitus, infection confirmed by appropriate culture, invasive mechanical ventilation and the mean of the following variables during postoperative ICU stay in both groups were registered: serum creatinine level, modification of diet in renal disease-glomerular filtration rate (MDRD-GFR), urea level, fluid balance, ejection fraction (EF), mean arterial blood pressure (MAP), infusion rate of inotropes or vasopressors, partial thromboplastin time (PTT), glucose level, and temperature.

\section{Whole blood viscosity measurement}

WBV is the intrinsic resistance of blood as it flows through blood vessels and is mainly determined by the shear rate of the flow, the volume fraction of red blood cells (hematocrit (Hct)), the concentration of plasma proteins namely fibrinogen, red blood cell (RBC) aggregation and red cell deformation [10, 23]. Viscosity can be represented as a function between shear rate and shear stress. Shear rate indicates the velocity of the blood flow and shear stress is the force of blood against 
the vessel wall. Fibrinogen has a greater influence on whole blood viscosity at low shear rates than at high shear rates due to fibrinogen induced RBC aggregation at low shear rates [24]. The interaction of fibrinogen and hematocrit on viscosity can be represented by an estimate of yield shear stress (YSS). YSS is the force required to start movement in a blood vessel [11, 22]. Furthermore, blood viscosity is dependent on temperature, especially at a temperature below $35{ }^{\circ} \mathrm{C}$ and above $39^{\circ} \mathrm{C}[25,26]$.

WBV was measured using the Contraves LS300 Low Shear Viscometer (ProRheo, Germany) within $180 \mathrm{~min}$ after blood sampling. The setting of the viscometer was standardized for all samples. Briefly, all blood tubes were placed on a shaker in the time between blood collection and the viscosity measurement. The viscosity was measured at $37 \pm 0.1{ }^{\circ} \mathrm{C}$, and at 23 different clinically relevant shear rate intervals $\left(0.02-50 \mathrm{~s}^{-1}\right)$ to minimize measurement errors [24]. As a measure for WBV, the area under the viscosity-shear rate curve (AUC) was used. The AUCs' between the shear rates 0.02 and $5 \mathrm{~s}^{-1}$ were adjusted for Hct since this has a major impact on blood viscosity at low shear rates [11, 24]. Adjustment was performed by dividing the blood viscosity between the shear rates 0.02 and $5 \mathrm{~s}^{-1}$ by Hct. This is an estimation of adjusted whole blood viscosity for Hct approximating the precise value [27]. In addition, the yield shear stress was analyzed to compare the influence of Hct and fibrinogen on WBV. YSS was calculated according to Equation $1[11,22]$.

$$
\mathrm{YSS}=13.5\left(10^{-6}\right) C_{f}^{2}(H c t-6)^{3}
$$

Where $\mathrm{C}_{\mathrm{f}}$ is the fibrinogen concentration in $\mathrm{mg} \%$.

\section{Statistical analysis}

We used a case:control ratio of $1: 4$, which resulted in a power of $94 \%$, with a two tailed alpha of 0.05 . Student's t-tests or Mann-Whitney $U$ tests were used depending on data distribution. The Chi-square test was used to test the dichotomous variables. Because of the high level of attrition Linear Mixed Model testing was used to study the association between blood viscosity, hematocrit, fibrinogen and delirium. A two tailed $p$ value of $<0.05$ was considered statistically significant. Statistical analysis was performed using IBM SPSS Statistics 20 and GraphPad Prism 5.0 (Graphpad Software, San Diego, CA, USA).

\section{Results}

In total 80 cardiothoracic surgical patients were screened. Of these, 16 (20\%) developed delirium postoperatively. One non-delirious patient was excluded due to serious complications and sustained coma, five non- delirious and four delirious patients were excluded because of missing data. Subsequently 12 cases were matched with 48 non-cases. Nine patients developed delirium within $24 \mathrm{~h}$, and 3 patients developed delirium within $72 \mathrm{~h}$ after surgery. Patient and demographic characteristics are depicted in Table 1.

\section{Postoperative levels of fibrinogen, hematocrit and whole blood viscosity}

Pre-operative fibrinogen, Hct and WBV were comparable between groups (Fig. 1). In both groups fibrinogen levels and hematocrit decreased significantly after surgery (both $p<0.001)$. No significant difference was found between both groups in reduction of fibrinogen $(p=0.36)$ and hematocrit $(p=0.23)$, Fig. 1. Postoperatively the AUC of WBV between shear rates 0.02 and $50 \mathrm{~s}^{-1}$ decreased significantly in both groups $(p<0.001)$. Again, there was no significant difference in this reduction between patients that developed delirium and those who did not $(p=0.80)$. The AUC of WBV between shear rates 0.02 and $5 \mathrm{~s}^{-1}$ remained similar over time in both groups and not different between both groups $(p=0.78)$ either. The AUC of blood viscosity adjusted for hematocrit was also comparable between the patients who developed delirium and those who did not $(p=0.33)$, (Figs. 1and 2). Finally, changes in the YSS were also not different between both groups $(p=0.68)$.

Changes in the Hct, fibrinogen, WBV and YSS did not differ significantly in patients that developed delirium within $24 \mathrm{~h}$ and those that developed delirium after $48 \mathrm{~h}$.

\section{Discussion}

We hypothesized that development of delirium in postcardiothoracic surgery patients could be related to changes in blood viscosity. In line with such a hypothesis is the fact that reduced cerebral blood flow has been suggested to be a possible pathway for the occurrence of delirium. In addition, strokes and dementia are correlated with occurrence of delirium. Both strokes and dementia have been associated with high blood viscosity $[28,29]$ and both occur more frequently after cardiothoracic surgery. In this exploratory case-control study we did not find differences in the changes in hematocrit, fibrinogen levels or whole blood viscosity in postcardiothoracic surgery patients who did or did not develop delirium. These findings indicate that changes in viscosity during the postoperative phase do not play a role in the development of post-operative delirium in cardiothoracic surgery patients. Even more so, the observed postoperative decrease of Hct and WBV provides no protection against delirium.

Although several studies have shown a strong correlation between whole blood viscosity and cerebral flow 
Table 1 Demographic variables of delirious and non-delirious patients

\begin{tabular}{|c|c|c|c|}
\hline Characteristic & Delirious $(N=12)$ & Non-delirious $(N=48)$ & $p$-value \\
\hline Age (years), (mean, SD) & $69(5.6)$ & $65(8.2)$ & 0.16 \\
\hline Male, N (\%) & $8(67 \%)$ & $36(75 \%)$ & 0.56 \\
\hline Duration of surgery in minutes (mean, SD) & $195(73)$ & $187(50)$ & 0.65 \\
\hline ECC-time (min), (mean, SD) & $107(43)$ & $90(21)$ & 0.21 \\
\hline AOX-time (min), (mean, SD) & $65(22)$ & $59.8(17)$ & 0.36 \\
\hline APACHE-\| Score (mean, SD) & $17(4)$ & $15(4)$ & 0.19 \\
\hline Euro score (mean, SD) & $3.8(2.0)$ & $3.5(2.0)$ & 0.68 \\
\hline Serum creatinine level ( $\mu$ mol. L-1), (median, IQR) & $87(68-94)$ & $81(73-94)$ & 0.72 \\
\hline MDRD-GFR (ml. min-1. 1.73 m-2), (median, IQR) & $78(59-90)$ & $81(64-90)$ & 0.49 \\
\hline Urea level (mmol. L-1), (median, IQR) & $8.0(7.2-9.1)$ & $7.3(6.0-8.6)$ & 0.20 \\
\hline Fluid balance (mL), (median, IQR) & $909(34-1171)$ & $375(-137-1121)$ & 0.65 \\
\hline EF (\%), (median, IQR) & $60.0(46.3-60.0)$ & $55.0(45.0-60.0)$ & 0.40 \\
\hline MAP (mmHg), (median, IQR) & $75(68-77)$ & $75(71-83)$ & 0.40 \\
\hline Admission of inotropes or vasopressors, N (\%) & $10(83 \%)$ & $28(58 \%)$ & 0.11 \\
\hline Duration of mechanical ventilation ( $\geq 24 \mathrm{~h}), \mathrm{N}(\%)$ & $0(0 \%)$ & $0(0 \%)$ & N/A \\
\hline PTT (sec), (median, IQR) & $19(18-21)$ & $18(18-19)$ & 0.26 \\
\hline Diabetes mellitus, N (\%) & $0(0 \%)$ & $5(10 \%)$ & 0.83 \\
\hline Glucose level (mmol. L-1), (median, IQR) & $7.9(7.3-8.8)$ & $7.6(6.7-8.2)$ & 0.36 \\
\hline Temperature $\left({ }^{\circ} \mathrm{C}\right)$, (median, IQR) & $36.6(35.3-37.1)$ & $36.6(35.9-36.9)$ & 0.93 \\
\hline Confirmed infection, N (\%) & $0(0 \%)$ & $0(0 \%)$ & N/A \\
\hline
\end{tabular}

Definition of abbreviations: ECC extracorporeal circulation, AOX aortic cross clamp, min minutes, APACHE II acute physiology and chronic health evaluation II, Euro European system for cardiac operative risk evaluation, IQR interquartile range, MDRD-GFR modification of diet in renal disease-glomerular filtration rate, $E F$ ejection fraction, MAP mean arterial blood pressure, N/A not applicable, PTT partial thromboplastin time, sec seconds, SD standard deviation

$[11,24]$, it appears plausible that due to low hematocrit levels after cardiothoracic surgery, the viscosity level is already so low, that moderate changes at this low level of blood viscosity do not affect cerebral blood flow. It has been shown, that a logarithmic correlation exists between hematocrit and whole blood viscosity, which is even stronger at lower shear rates [30]. Another reason why our hypothesis was not confirmed could be, that cerebral blood flow and delirium are not strongly correlated. However, earlier studies have demonstrated a correlation between decreased cerebral blood flow and delirium $[9,10]$.

The postoperative changes in WBV we observed are in accordance with previous reports in cardiothoracic surgery patients $[12,31]$. However, in those studies, viscocity was only measured at a high shear rate $\left(90 \mathrm{~s}^{-1}\right)$, while in our study we measured viscosity at 23 different and at low shear rates. We found no postoperative changes in WBV at shear rates between 0.02 and $5 \mathrm{~s}^{-1}$ in cardiothoracic surgery patients. At low shear rates, red blood cells clump together due to fibrinogen induced RBC aggregation resulting in a higher viscosity which is dependent on both Hct and fibrinogen concentration [11, 24]. Therefore, unlike Papp et al. [12], we took a small part of the blood viscosity curve at low shear rates (between 0.02 and $5 \mathrm{~s}^{-1}$ ) and corrected for hematocrit. Nevertheless, there was no significant difference between delirious and non-delirious patients.

Although duration of delirium is not a true reflection of severity of delirium, this is in several studies used as a measure for delirium severity. However, in our study the maximum delirium duration was 3 days (1 patient) and the rest had 1-2 days of delirium. For this reason we have treated delirium as a dichotomous variable. Data from other studies show that the median duration of delirium in cardiac surgery patients is two days [3, 32]. This is comparable to our data.

Some limitations of this study need to be considered. Firstly, the gold standard to diagnose delirium was not used. The gold standard is clinical research, performed by a psychiatrist, a neuropsychologist or a geriatrician. Delirium fluctuates during the day, therefore the gold standard is not always practical [33]. Instead of the gold standard, the internationally validated CAM-ICU and DOS scale performed by nurses were used to enable multiple assessments per day per patient [19]. At each nursing shift, patients were screened for delirium. Using multiple 


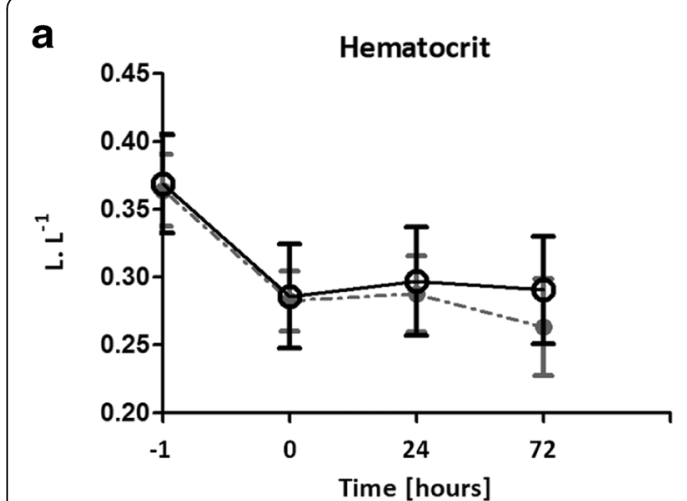

C Blood viscosity AUC between the shear rates 0.02

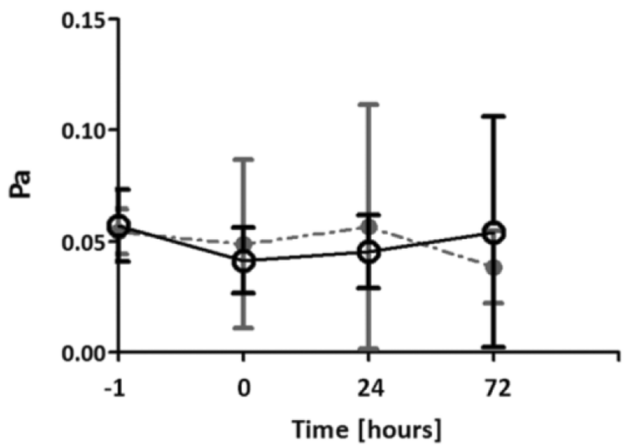

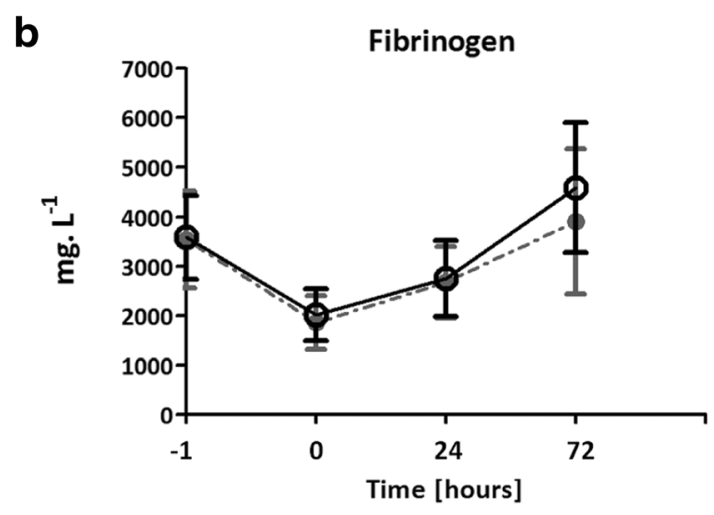

d Blood viscosity AUC between the shear rates 0.02 and $50 \mathrm{~s}^{-1}$

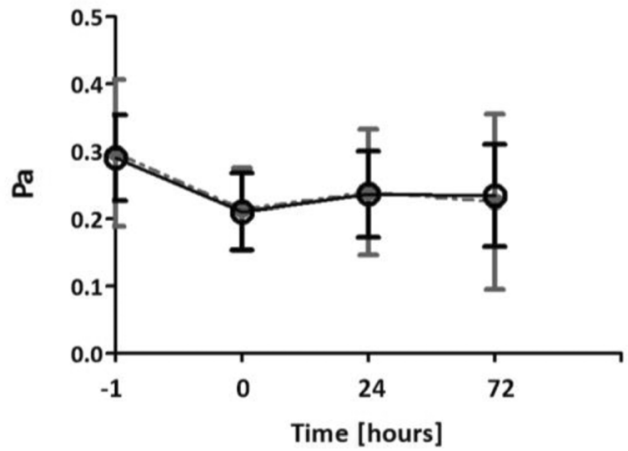

\section{e Blood viscosity AUC corrected for hematocrit}

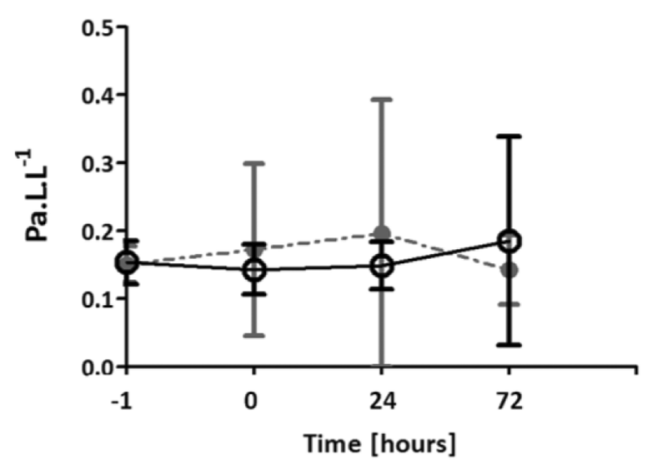

Fig. 1 Postoperative levels of hematocrit, fibrinogen and whole blood viscosity of delirious and non-delirious patients. a Hematocrit. b Fibrinogen. c The area under curve (AUC) of the whole blood viscosity (WBV) between shear rates 0.02 and $5 \mathbf{s}^{-1}$. $\mathbf{d}$ The area under curve (AUC) of the whole blood viscosity (WBV) between shear rates 0.02 and $50 \mathrm{~s}^{-1}$. e The area under curve (AUC) of the whole blood viscosity corrected for hematocrit. T. 1, directly after the induction of anesthesia. $T_{0}$, within one hour of Intensive Care Unit admission. $T_{24}$, one day after cardiothoracic surgery. $T_{72}$, three days after cardiothoracic surgery. Data are expressed as mean and SD. Linear Mixed Model testing was used to determine a difference between the two groups. No significant differences were found. A two tailed $p$ value of $<0.05$ was considered statistically significant

assessments per day increases the sensitivity of delirium diagnosis. In addition, in order to minimize under-diagnosis, the reports of the doctors and nurses were analyzed for indications of delirium in combination with the use of anti-psychotics. Secondly, in this study we included both the CABG as well as the aortic valve surgery patients. In comparison to closed heart surgery, patients undergoing open heart surgery have an increased risk for cerebral embolization $[34,35]$. Although, the latter introduces heterogeneity, it results in data that can be generalized more easily to the daily practice of cardiac surgery. Finally, in this study $25 \%$ of the data is missing. However, we used Linear Mixed Models testing in which it is allowed to have $25-30 \%$ of missing [36]. 

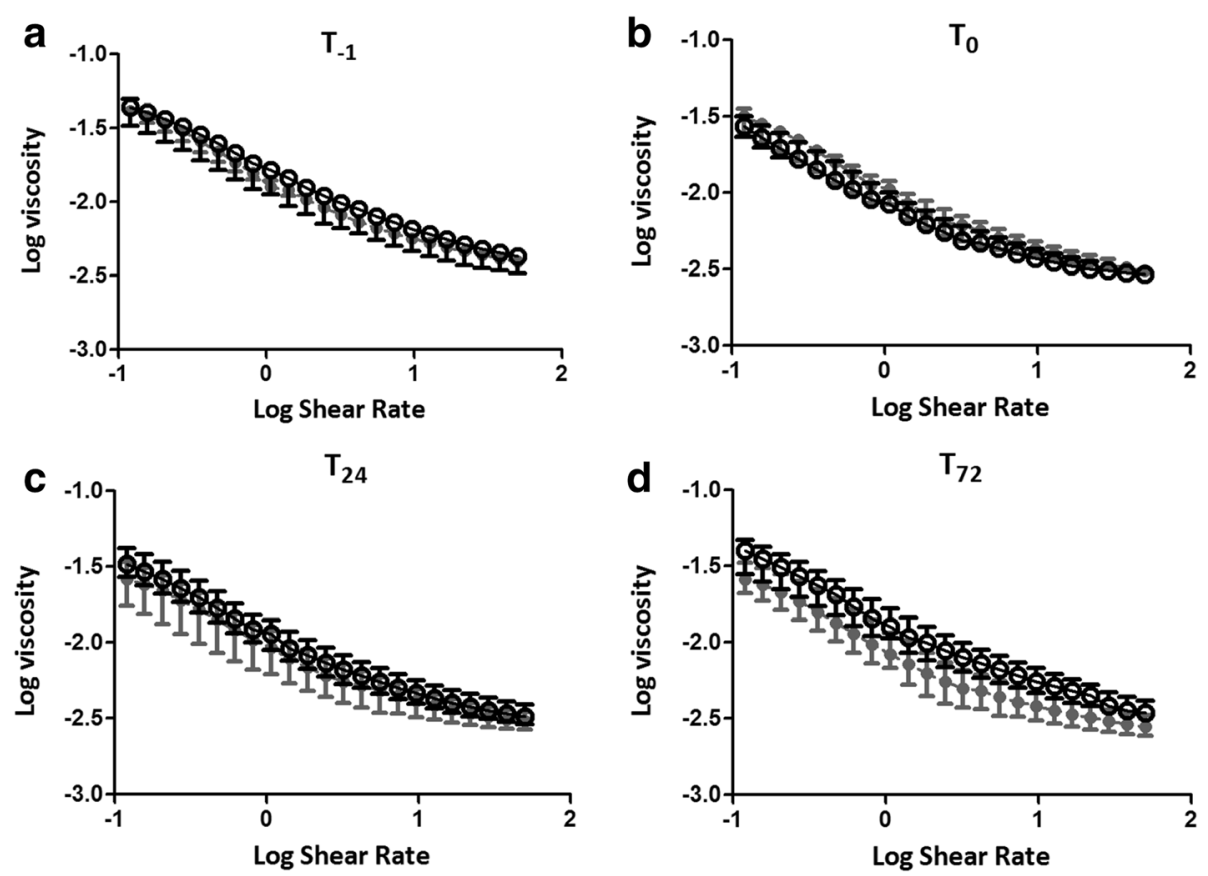

Fig. 2 Whole blood viscosity at 23 different shear rates between 0.02 and $50 \mathrm{~s}^{-1}$ of delirious and non-delirious patients. Log 10 of whole blood viscosity versus $\log 10$ of shear rates. $\mathbf{a}$ Directly after the induction of anesthesia $\left(T_{-1}\right)$. b Within one hour of Intensive Care Unit admission $\left(T_{0}\right)$. C One day after cardiothoracic surgery $\left(T_{24}\right)$. d Three days after cardiothoracic surgery $\left(T_{72}\right)$

\section{Conclusion}

In this group of cardiothoracic surgery patients no association was found between whole blood viscosity and the development of post-operative delirium. This finding indicates that in postoperative cardiothoracic surgery patients, delirium is probably not related to blood viscosity changes.

\section{Abbreviations}

AOX, aortic cross clamp; APACHE, acute physiology and chronic health evaluation; AUC, area under the curve; CABG, coronary artery bypass grafting; CAM-ICU, confusion assessment method intensive care unit; $C_{f}$ fibrinogen concentration; DOS, delirium screening observation; ECC, extracorporeal circulation; EF, ejection fraction; Euro score, European system for cardiac operative risk evaluation; $\mathrm{Hct}$, hematocrit; ICU, intensive care unit; IQR, interquartile range; MAP, mean arterial blood pressure; MDRD-GFR, modification of diet in renal disease-glomerular filtration rate; N/A, not applicable; PTT, partial thromboplastin time; RBC, red blood cell; SD, standard deviation; WBV, whole blood viscosity; YSS, yield shear stress.

\section{Acknowledgment}

The authors would like to thank the nurses on the intensive care and medium care unit and cardiothoracic surgical ward for their help with the blood collections. They also would like to thank prof. dr. ir. Robert F. Mudde and L. Bergwerff MSC for their help with analysis of the data. Dr WF Abdo received financial support from the Netherlands Organization for Health Research and Development (ZonMW Clinical Fellowship number 90715610)

\section{Authors' contributions}

All authors participated in design of the study, collection of the data, analysis of the data and critically reviewed the paper. All authors read and approved the final manuscript and gave consent for publication.

\section{Competing interests}

The authors declare that they have no competing interests.

\section{Author details}

${ }^{1}$ Department of Intensive Care Medicine, Radboudumc, Nijmegen, The Netherlands. ${ }^{2}$ Department of Cardiothoracic Surgery, Radboudumc, Nijmegen, The Netherlands. ${ }^{3}$ Department of Cardiology, Radboudumc, Nijmegen, The Netherlands. ${ }^{4}$ Department of Intensive Care Medicine, University Medical Centre Utrecht, Utrecht, The Netherlands.

Received: 8 December 2015 Accepted: 27 July 2016

Published online: 05 August 2016

\section{References}

1. van den Boogaard M, Schoonhoven $L$, Maseda E, Plowright $C$, Jones $C$, Luetz A, Sackey PV, Jorens PG, Aitken LM, van Haren FM, Donders R, van der Hoeven JG, Pickkers P. Recalibration of the delirium prediction model for ICU patients (PRE-DELIRIC): a multinational observational study. Intensive Care Med. 2014;40(3):361-9. doi:10.1007/s00134-013-3202-7.

2. Dubois MJ, Bergeron N, Dumont M, Dial S, Skrobik Y. Delirium in an intensive care unit: a study of risk factors. Intensive Care Med. 2001;27(8):1297-304.

3. van den Boogaard M, Schoonhoven L, van der Hoeven JG, van Achterberg T, Pickkers P. Incidence and short-term consequences of delirium in critically ill patients: a prospective observational cohort study. Int J Nurs Stud. 2012;49(7):775-83. doi:10.1016/j.ijnurstu.2011.11. 016. Epub 2011 Dec 22.

4. Koster S, Hensens AG, Schuurmans MJ, van der Palen J. Risk factors of delirium after cardiac surgery: a systematic review. Eur J Cardiovasc Nurs. 2011;10(4):197-204. doi:10.1016/j.ejcnurse.2010.09.001. Epub 2010 Sep 25.

5. Li HC, Chen YS, Chiu MJ, Fu MC, Huang GH, Chen CC. Delirium, Subsyndromal Delirium, and Cognitive Changes in Individuals Undergoing Elective Coronary Artery Bypass Graft Surgery. J Cardiovasc Nurs. 2015;30(4): 340-5. doi:10.1097/JCN.0000000000000170.

6. Krähenbühl ES, Immer FF, Stalder M, Englberger L, Eckstein FS, Carrel TP. Temporary neurological dysfunction after surgery of the thoracic aorta: a predictor of poor outcome and impaired quality of life. Eur J Cardiothorac Surg. 2008;33(6):1025-9. doi:10.1016/j.ejcts.2008.01.058. Epub 2008 Mar 17. 
7. Van Rompaey B, Schuurmans MJ, Shortridge-Baggett LM, Truijen S, Bossaert L. Risk factors for intensive care delirium: a systematic review. Intensive Crit Care Nurs. 2008;24(2):98-107. Epub 2007 Oct 18.

8. Flacker JM, Lipsitz LA. Neural mechanisms of delirium: current hypotheses and evolving concepts. J Gerontol A Biol Sci Med Sci. 1999;54(6):B239-46.

9. Gunther ML, Morandi A, Ely EW. Pathophysiology of Deliriumin in the Intensive Care Uni. Crit Care Clin. 2008;24(1):45-65. viii.

10. Yokota H, Ogawa S, Kurokawa A, et al. Regional cerebral blood flow in delirium patients. Psychiatry Clin Neurosci. 2003;57(3):337-9.

11. Grotta J, Ackerman R, Correia J, Fallick G, Chang J. Whole blood viscosity parameters and cerebral blood flow. Stroke. 1982;13:296-301 doi:10.1161/01.

12. Papp J, Toth A, Sandor B, Kiss R, Rabai M, Kenyeres P, et al. The influence of onpump and off-pump coronary artery bypass grafting on hemorheological parameters. Clin Hemorheol Microcirc. 2011;49(1-4):331-46.

13. Papaioannou TG, Stefanadis C. Vascular Wall Shear Stress: Basic Principles and Methods. Hellenic J Cardiol. 2005;46:9-15.

14. Cipolla JM. The cerebral circulation. University of Vermont College of Medicine. San Rafael (CA): Morgan \& Claypool Life Sciences; 2009.

15. Craver JM, Bufkin BL, Weintraub WS, Guyton RA. Neurologic events after coronary bypass grafting: further observations with warm cardioplegia. Ann Thorac Surg. 1995;59(6):1429-33. discussion 1433-4.

16. Ruggiero HA, Castellanos H, Caprissi LF, Caprissi ES. Heparin effect on blood viscosity. Clin Cardiol. 1982;5(3):215-8.

17. Czerny $M$, Krähenbühl $E$, Reineke $D$, Sodeck $G$, Englberger $L$, Weber $A$, Schmidli J, Kadner A, Erdoes G, Schoenhoff F, Jenni H, Stalder M, Carrel T. Mortality and neurologic injury after surgical repair with hypothermic circulatory arrest in acute and chronic proximal thoracic aortic pathology: effect of age on outcome. Circulation. 2011;124(13):1407-13. doi:10.1161/CIRCULATIONAHA.110.010124. Epub 2011 Aug 29.

18. Ely EW, Margolin R, Francis J, May L, Truman B, Dittus R, et al. Evaluation of delirium in critically ill patients: validation of the Confusion Assessment Method for the Intensive Care Unit (CAM-ICU). Crit Care Med. 2001:29(7):1370-9.

19. Schuurmans MJ, Shortridge-Baggett LM, Duursma SA. The Delirium Observation Screening Scale: a screening instrument for delirium. Res Theory Nurs Pract. 2003;17(1):31-50.

20. Gusmao-Flores D, Salluh JIF, Chalhub RÁ, Quarantini LC. The confusion assessment method for the intensive care unit (CAM-ICU) and intensive care delirium screening checklist (ICDSC) for the diagnosis of delirium: a systematic review and meta-analysis of clinical studies. Crit Care. 2012;16(4): R115. 10.1186/cc11407.

21. Inouye SK, Leo-summers ÃL, Zhang Y, Bogardus ST, Leslie DL, Agostini JV. A Chart-Based Method for Identification of Delirium : Validation Assessment Method. J Am Geriatr Soc. 2005:53(2):312-8.

22. Merrill EW, Cheng CS, Pelletier GA. Yield Stress of Normal Human Blood as a Function of Endogenous Fibrinogen. J Appl Physiol. 1969;26(1):1-3.

23. Fong TG, Bogardus ST, Daftary A. Interrelationship between delirium and dementia:cerebral perfusion changes in older delirious patients using 99mTc HMPAO SPECT. J Gerontol A Biol Sci Med Sci. 2006;61A:1294-9.

24. Pop G, Bisschops LL, Iliev B, Struijk PC, van der Hoeven JG, Hoedemaekers $\mathrm{CW}$. On-line blood viscosity monitoring in vivo with a central venous catheter, using electrical impedance technique. Biosens Bioelectron. 2013; 41:595-601. doi:10.1016/j.bios.

25. Ayres ML, Jarrett PEM, Browse NL. Blood viscosity, Raynaud's phenomenon and the effect of fibrinolytic enhancement. Br J Surg. 1981;68(1):51-4.

26. Cinar $Y$, Senyol AM, Duman K. Blood viscosity and blood pressure: role of temperature and hyperglycemia. Am J Hypertens. 2001;14(5 Pt 1):433-8.

27. Matrai A, Whittington RB, Ernst E. A simple method of estimating whole blood viscosity at standardized hematocrit. Clin Hemorheol. 1987;7:261-5.

28. Park M-S, Kim B-C, Kim I-K, Lee S-H, Choi S-M, Kim M-K, Lee S-S, Cho K-H. Cerebral Infarction in IgG Multiple Myeloma with Hyperviscosity. J Korean Med Sci. 2005;20(4):699-701. doi:10.3346/jkms.2005.20.4.699.

29. Grigg AP, Allardice J, Smith IL, Murray W, Horsfall D, Parkin D. Hyperviscosity syndrome in disseminated breast adenocarcinoma. Pathology. 1994;26(1):65-8.

30. Aronson HB, Cotev S, Magora F, Borman JB, Merin G. Blood viscosity and open heart surgery. A comparison of values in systemic and pulmonary blood vessels. Br J Anaesth. 1974;46(10):722-5.

31. Weaver JP, Evans A, Walder DN. The effect of increased fibrinogen content on the viscosity of blood. Clin Sci. 1969;36(1):1-10.

32. Koster S, Oosterveld FG, Hensens AG, Wijma A, van der Palen J. Delirium after cardiac surgery and predictive validity of a risk checklist. Ann Thorac Surg. 2008;86(6):1883-7. doi:10.1016/j.athoracsur.2008.08.020.
33. American Psychiatric Association. Diagnostic and Statistical Manual of Mental Disorders (DSM-IV). 4th ed. Washington D.C: American Psychiatric Association; 1994

34. Wolman RL, Nussmeier NA, Aggarwal A, Kanchuger MS, Roach GW, Newman MF, Mangano CM, Marschall KE, Ley C, Boisvert DM, Ozanne GM, Herskowitz A, Graham SH, Mangano DT. Cerebral injury after cardiac surgery: identification of a group at extraordinary risk. Multicenter Study of Perioperative Ischemia Research Group (McSPI) and the Ischemia Research Education Foundation (IREF) Investigators. Stroke. 1999;30(3):514-22.

35. Ying Tan M, Amoako D. Postoperative cognitive dysfunction after cardiac surgery. Contin Educ Anaesth Crit Care Pain. 2013. doi: 10.1093/bjaceaccp/ mkt022.

36. Twisk JWR, Ellenberg SS, Elston R, Everitt B, Everitt BS, Harrell F. Applied Multilevel Analysis: A Practical Guide for Medical Researchers (Practical Guides to Biostatistics and Epidemiology). ISBN10-0521614988, ISBN139780521614986

\section{Submit your next manuscript to BioMed Central and we will help you at every step:}

- We accept pre-submission inquiries

- Our selector tool helps you to find the most relevant journal

- We provide round the clock customer support

- Convenient online submission

- Thorough peer review

- Inclusion in PubMed and all major indexing services

- Maximum visibility for your research

Submit your manuscript at www.biomedcentral.com/submit

Biomed Central 